\title{
$\mathbf{N}$ 型多孔硅/镍微米管复合材料的制备及其磁性能研究
}

\author{
周 辉, 韩满贵, 唐中开, 吴燕辉 \\ (电子科技大学 电子薄膜与集成器件国家重点实验室, 国家电磁辐射控制材料工程技术研究中心, 成都 610054)
}

摘 要: 通过背光照辅助电化学腐蚀的方法, 在 $\mathrm{N}$ 型轻掺杂的(100)单晶硅上制备了多孔硅模板。模板孔端口呈类正 方形结构, 孔边长约为 $2 \mu \mathrm{m}$, 孔深约 $50 \mu \mathrm{m}$ 。然后在制备的 $\mathrm{N}$ 型多孔硅模板中电沉积制备了磁性镍微米管。与绝 缘性 $\mathrm{AAO}$ 模板中金属从孔底部开始生长不同，在半导体性质的 $\mathrm{N}$ 型多孔硅模板中，镍沿着孔壁均匀生长，并随着 沉积时间的延长，由颗粒状逐渐生长为微米管，镍的晶型没有发生变化。由于镍微米管的缺陷和晶界比镍颗粒更少， 畴壁位移更容易, 所以具有更小的矫顽力。同时受强烈的退磁能影响，外加磁场在垂直和平行镍微米管方向时测得 的磁滞回线表现出明显的磁各向异性。

关 键 词: $N$ 型多孔硅; 电化学沉积; 镍微米管; 磁各向异性

中图分类号: TQ153 文献标识码: A

\section{Fabrication and Magnetic Properties of N-type Porous Silicon/Nickel Microtubes Composite}

\author{
ZHOU Hui, HAN Man-Gui, TANG Zhong-Kai, WU Yan-Hui
}

\begin{abstract}
(State Key Laboratory of Electronic Thin Films and Integrated Devices, National Engineering Research Center of Electromagnetic Radiation Control Materials, University of Electronic Science and Technology, Chengdu 610054, China)
\end{abstract}

\begin{abstract}
The highly ordered n-type porous Si template was prepared via electrochemical etching and back side illumination method. Pores of the Si template had squarish shape with side length of about $2 \mu \mathrm{m}$ and depth of about $50 \mu \mathrm{m}$, respectively. Three growth stages, nucleation, self-assembly, and stable growth were observed by scanning electron microscope (SEM) during the formation process of the porous n-silicon template. Subsequently, nickel microtubes arrays were synthesized by electro-deposition. And the formation mechanism of the microtubes was also investigated. The content and particle size of Ni particles embedded in n-type porous Si templates increase with the increase of deposition time. Ni microtubes are formed by gathering the Ni particles over pore sidewall, which is different from that of insulative AAO template. In addition, the crystal structure of Ni microtubes keeps fcc-Ni in the whole growth process. In particular, due to the fewer defects and grain boundaries of Ni microtubes than that of $\mathrm{Ni}$ particles, the coercivity value of Ni microtubes is smaller. Moreover, the magnetic anisotropy of Ni microtubes is mainly contributed to the strong demagnetization energy.
\end{abstract}

Key words: n-type porous silicon; electrochemical deposition; Ni microtubes; magnetic anisotropy

近年来，一维磁性材料由于在微波器件、磁存 储和传感器等领域具有潜在的应用价值而得到广泛
关注 ${ }^{[1-4]}$ 。利用电化学沉积法在 AAO 模板上制备磁 性纳米线或纳米管已成为成熟且普遍的方式 ${ }^{[5-7]}$ 。

收稿日期: 2015-11-30; 收到修改稿日期：2016-03-11

基金项目：国家自然科学基金重点项目(61271039); 四川省科技项目(2015HH0016)

National Natural Science Foundation of China(61271039); Scientific Projects of Sichuan Province(2015HH0016)

作者简介: 周 辉(1990-), 男, 硕士研究生. E-mail: 704196761@qq.com

通讯作者: 韩满贵, 教授. E-mail: han_mangui@yahoo.com. 
但是, AAO 模板容易破裂、不耐酸碱腐蚀, 且孔径 只能控制在 10 300 $\mathrm{nm}$ 的纳米级范围 ${ }^{[8]}$, 这些不足 限制了其实际应用。多孔硅的机械强度高、耐化学 腐蚀, 且孔径可在纳米到微米范围内调节, 并且硅 是最常见的半导体材料, 可以很好地与半导体工艺 相结合，大大方便了其应用。

1990 年, Lehmann 等 ${ }^{[9]}$ 就采用背光照法制备多 孔硅模板。背光照方法就是通过加背面光照并利用 光刻技术、聚苯乙烯小球和离子束刻蚀等方法在 $\mathrm{N}$ 型 $\mathrm{Si}(100)$ 表面预压印一层排列规整的图案, 进而在 预成核的基础之上进行电化学腐蚀, 得到有序度非 常高的孔形貌 ${ }^{[10-15]}$ 。迄今为止, 此法一直是制备高 质量多孔硅模板的最佳选择之一 ${ }^{[16-17]}$, 但是其中表 面预压印的方法成本太高。为了节约生产成本, 采 用电化学随机腐蚀法来制备多孔硅模板, 利用高电 压预成核方法，可以避免复杂的预压印成核技术。

近年来, 有学者利用纳米级多孔硅制备一维纳 米磁性材料 ${ }^{[18-27]}$ 。Cheng 等 ${ }^{[28]}$ 和 Kazuhiro 等 ${ }^{[29]}$ 分别 在 $\mathrm{N}$ 型和 $\mathrm{P}$ 型微米级多孔硅中电沉积制备了 $\mathrm{Cu}$ 、 $\mathrm{Pt} 、 \mathrm{Pd}$ 和 $\mathrm{Au}$ 等贵金属, 发现它们都是从孔底部开 始生长并生成微米棒状结构。Katsutoshi 等 ${ }^{[30]}$ 发现 在不加背光照条件下, $\mathrm{Cu}$ 可在 $\mathrm{P}$ 型多孔硅中正常生 长为微米棒, 而 $\mathrm{Ni}$ 的沉积必须施加背光照, 围绕着 孔壁开始生长并最终形成微米管。然而, 在 $\mathrm{N}$ 型多孔 硅中沉积镍的微米管状结构的研究仍鲜有报道 ${ }^{[31]}$ 。

因此, 本工作将研究磁性金属材料 $\mathrm{Ni}$ 在 $\mathrm{N}$ 型微米级 多孔硅中的沉积, 并探究镍的生长过程以及生长机 制, 对其结构变化和磁性能进行详细的探讨。

\section{1 实验方法}

\section{1 仪器、材料与试剂}

仪器: CHI660C 电化学工作站(上海辰华仪器有 限公司)、X 射线衍射仪(SHIMADZU, XRD-7000)、 场发射扫描电子显微镜(JEOL, JSM-7600F)、振动样 品磁强计(ModelBHV-525)、电子束蒸发(成都启合真 空设备有限公司)。

材料：单面抛光的 $\mathrm{N}$ 型(100)硅基片。

试剂: 氢氟酸、乙醇、丙酮、 $\mathrm{NiCl}_{2} \cdot 6 \mathrm{H}_{2} \mathrm{O}$ 和 $\mathrm{H}_{3} \mathrm{BO}_{3}$ 等试剂均为分析纯级别。

\section{2 多孔硅模板的制备}

采用单面抛光的 $\mathrm{N}$ 型(100)单晶硅片 (电阻率为 0.5 0.9 $\Omega \cdot \mathrm{cm}$ ), 利用电化学随机腐蚀法制备多孔硅 模板。电化学腐蚀过程在自制的反应槽中进行。

第一步: 清洗硅片, 然后利用电子束蒸发在硅
片未抛光面的边缘镀上铜导电层, 这样既能与铜反 应电极形成欧姆接触, 又不影响对硅片未抛光面的 光照(以后简称背光照)。

第二步: 安装好电化学反应装置。采用电化学 工作站提供电源, 参比电极为饱和甘录电极 (SCE), 对电极为铂电极, 保持硅片抛光面与反应槽内的腐 蚀液接触, 硅片未拋光面的边缘镀铜区域与铜反应 电极接触。

第三步: 配置 HF 酸腐蚀液。腐蚀液由氢氟酸、 去离子水和乙醇溶液组成, 其中氢氟酸浓度为 $6 \mathrm{wt} \%$, 并保持去离子水和乙醇的体积相等。

第四步: 将腐蚀液倒入反应槽内, 采用 $50 \mathrm{~W}$ 的 甾素光灯为硅片提供背光照, 并保持光源与硅片的 距离不变。

第五步: 进行电化学腐蚀反应。先施加 $10 \mathrm{~V}$ 的 电压反应 $3 \mathrm{~min}$, 再加 $0.15 \mathrm{~V}$ 的电压反应 $80 \mathrm{~min}$ 。 反应过程中对腐蚀液施加均匀搅拌, 待反应结束后 取出硅片并清洗干净, 利用扫描电镜观察多孔硅模 板形貌。

\section{3 电化学沉积镍}

采用制备好的多孔硅模板进行电化学沉积反应 制备镍微米管。电化学沉积与电化学腐蚀反应使用 相同的电源和反应槽。

第一步: 使用粒径约为 $7 \mu \mathrm{m}$ 的氧化铝研磨粉磨 去多孔硅表面几微米左右孔洞较无序的成核层, 露出 下面稳定生长阶段的深孔，因为不需要背光照，可将 研磨好的多孔硅基片未抛光面整体镀上铜导电层。

第二步：反应装置安装以及电源线的连接与电 腐蚀反应一样。

第三步：配置 $0.2 \mathrm{~mol} / \mathrm{L}$ 的 $\mathrm{NiCl}_{2} \cdot 6 \mathrm{H}_{2} \mathrm{O}$ 和 $\mathrm{H}_{3} \mathrm{BO}_{3}$ 混合液作为电解液, 将溶液倒入反应槽侵泡 $2 \mathrm{~h}$ 以 上, 以使溶液充分渗入多孔硅模板的深孔内。

第四步: 沉积电压为 $-1.5 \mathrm{~V}$, 沉积过程在室温下 进行并对溶液加以摚拌, 分别沉积 5、20、40 和 $90 \mathrm{~min}$, 观察镍的生长形貌。将制备好的基片先后用去离子 水和无水乙醇清洗数次, 最后用氮气吹干。

\section{2 结果与讨论}

\section{1 多孔硅模板的形貌与生长机理}

制备的多孔硅模板的 SEM 形貌如图 1 所示。由 图 1(a)可知，横断面形貌可分为三种不同的区域， 标识为 I 、 II 、III, 分别对应三个生长阶段：成核阶 段、自组装阶段和稳定生长阶段 ${ }^{[32]}$ ，三个区域没有 明确的界限(如图 1(a))。使用约 $7 \mu \mathrm{m}$ 的氧化铝研磨 

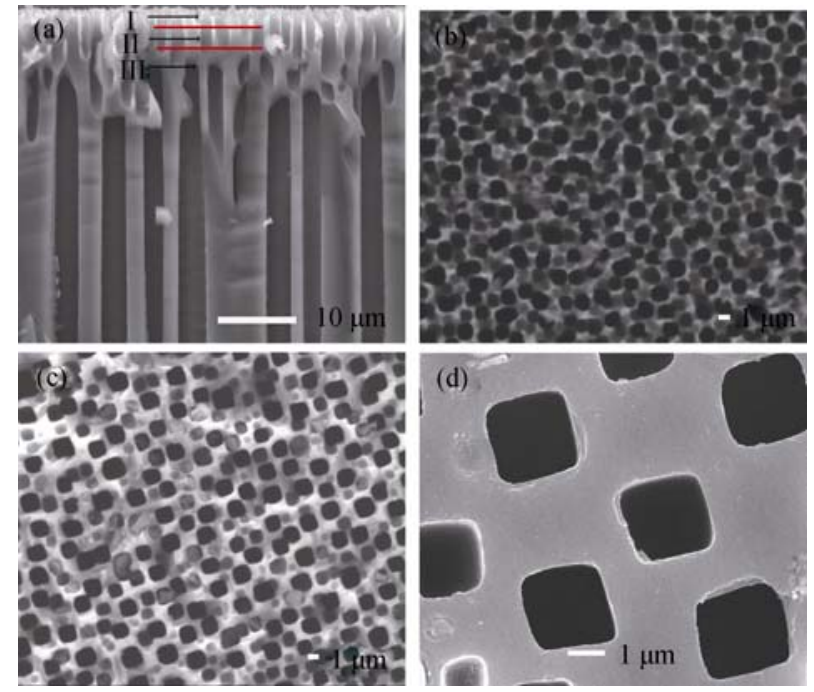

图 1 多孔硅模板的 SEM 照片

Fig. 1 Typical SEM images of porous silicon template (a) Cross section image of porous silicon; The front side of (b) region I, (c) region II, (d) region III in the Fig. 1(a)

粉对多孔硅模板正面研磨不同程度, 来获取三个生 长阶段的形貌特征。如图 1(b d ) 分别展现了三个生 长区域对应的模板正面形貌。没有预成核时, 形成 有序大孔较困难, 经过成核阶段和自组装阶段后, 进入微米孔洞的稳定生长阶段。在成核阶段(如图 1(b)), 硅片表面将会在氢氟酸和空穴的作用下产生 随机分布的小孔。实验采用高电压预腐蚀的方法, 通过加高电压产生大电流来促进成核; 在自组装阶 段(如图 1(c)), 一些孔会停止生长, 而另一些孔会继 续生长, 两个或者更多的小孔合并成一个大孔进入 稳定生长阶段而产生深孔; 在稳定生长阶段(如图 1(d)), 大孔将继续生长, 孔端口近似为正方形, 孔 边长约为 $2 \mu \mathrm{m}$, 孔深约为 $50 \mu \mathrm{m}$ 。

\section{2 镍微米管的形貌与结构特征}

为了研究镍的生长过程和生长机制, 分别对 沉积 $5 \mathrm{~min} 、 20 \mathrm{~min} 、 40 \mathrm{~min} 、 90 \mathrm{~min}$ 的样品形貌 进行了 SEM 表征(如图 2 所示)。沉积 $5 \mathrm{~min}$ 时, 镍 以颗粒的形式沿孔壁生长, 此时颗粒数量很少; 沉 积 $20 \mathrm{~min}$ 时, 围绕着孔壁生长的镍颗粒逐渐增多, 但是还没有完全包围孔壁; 沉积 $40 \mathrm{~min}$ 时, 镍不再 是分立的颗粒, 而是连成一片包围了整个孔壁, 形 成清晰的管状结构, 壁厚约为 $(320 \pm 50) \mathrm{nm}$ 。另外, 从各个时间段的样品正面图可以清晰地看到由镍 颗粒逐渐生长为镍微米管的过程; 沉积时间达到 $90 \mathrm{~min}$ 时, 由图 2(d) 可知, 多孔硅表面沉积了一层 较厚的磁性镍薄膜, 这是由于在微米管形成以后 继续延长沉积时间, 镍会在已沉积的镍上进行沉 积, 从而堵塞孔洞 ${ }^{[28-29]}$ 。
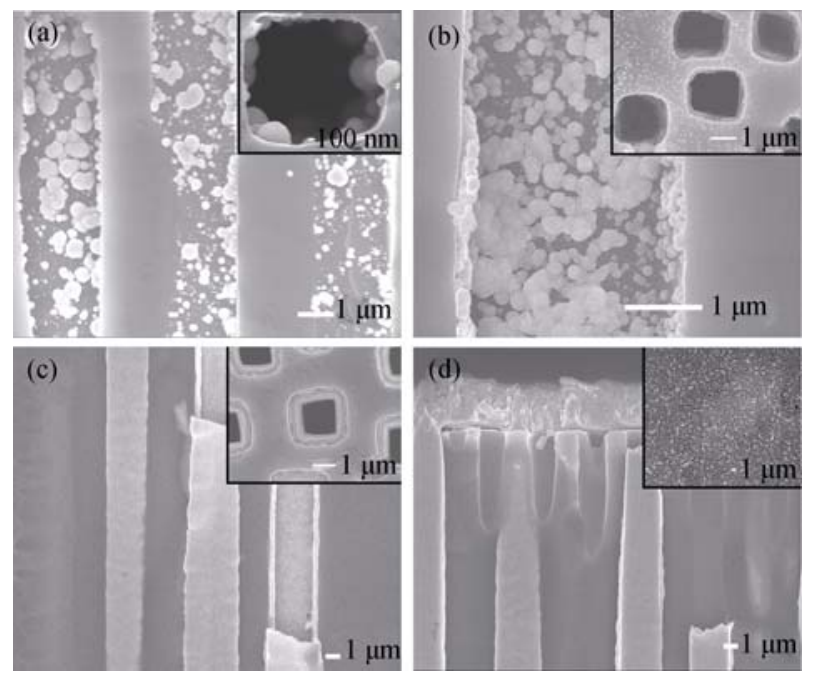

图 2 电化学沉积不同时间的镍颗粒与镍微米管的横截面和 正面的 SEM 照片

Fig. 2 SEM images of the cross section and front side of $\mathrm{Ni}$ particles and microtubes with different electrodeposition time (a) $5 \mathrm{~min}$; (b) $20 \mathrm{~min}$; (c) $40 \mathrm{~min}$; (d) $90 \mathrm{~min}$. The insert in each image is the front side of the template

对于 $\mathrm{AAO}$ 模板, 由于氧化铝的绝缘性, 电化学 沉积金属材料时, 金属颗粒将从 AAO 模板纳米孔 洞底部的导电层开始生长而不是孔壁 ${ }^{[6,33]}$ 。但由本 实验的生长过程可知, 镍并不是从多孔硅模板孔底 部而是围绕着孔壁开始生长, 这可归功于 $\mathrm{N}$ 型硅半 导体的导电性质。在 $\mathrm{N}$ 型多孔硅中, 电子是多数载 流子, 沉积反应在电流驱动下自动进行, 所以镍会 围绕着孔壁生长 ${ }^{[30]}$ 。

为了进一步分析沉积物质的结构和组成, 分 别对多孔硅模板及沉积 5、20、40 和 $90 \mathrm{~min}$ 的样 品进行了 X 射线衍射分析, 结果如图 3 所示。沉积 $\mathrm{Ni}$ 的四个样品均能检测到相同的结晶相。XRD 图 谱上三个明显的衍射峰表明生成的物相为面心立 方 $\mathrm{Ni}$ 的晶体结构(Space Group: Fm-3m; JCPDS 4-850), 且三个峰分别对应(111)、(200)和(220)三个 晶面。随着沉积时间的延长, 镍颗粒数量增加, 衍 射峰强度增大。

\section{3 镍微米管的磁性能}

图 4 为沉积 $5 \mathrm{~min}$ 的镍颗粒和沉积 $40 \mathrm{~min}$ 的镍 微米管的磁滞回线, 曲线(a)和(b)分别为外加磁场 平行 $\left(H_{\text {/ }}\right)$ 于孔轴线方向的镍颗粒与镍微米管的磁 滞回线。由图 2 可知, 大部分的镍颗粒的尺寸范围 为 $50 \mathrm{~nm} \sim 500 \mathrm{~nm}$ 之间, 远大于镍单畴颗粒尺寸 ${ }^{[34]}$ 。从 曲线(a)和(b)可以看出, 镍颗粒的矫顽力为 $11300 \mathrm{~A} / \mathrm{m}$, 大于微米管的矫顽力 $9390 \mathrm{~A} / \mathrm{m}$ 。这是由于在形成微 米管之前体系的缺陷和晶界更多, 畴壁位移更难, 导致矫顽力更大 ${ }^{[22]}$ 。 


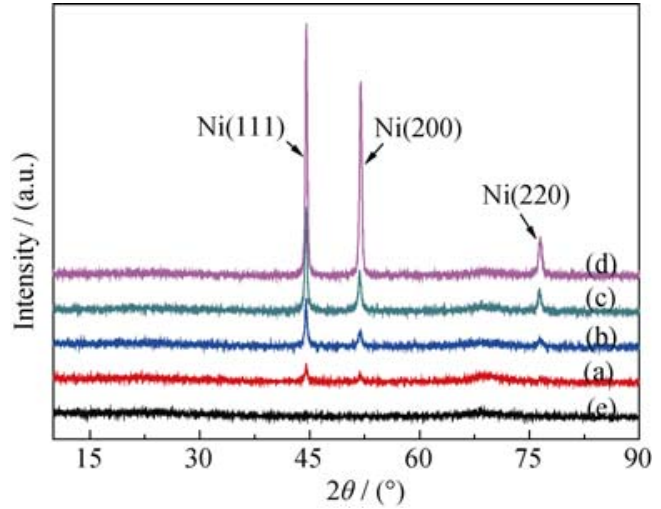

图 3 沉积不同时间的镍颗粒与镍微米管的 XRD 图谱

Fig. 3 XRD patterns of Ni particles and microtubes deposited for different time

(a) $5 \mathrm{~min}$; (b) $20 \mathrm{~min}$; (c) $40 \mathrm{~min}$; (d) $90 \mathrm{~min}$; (e) n-type porous silicon template

图 4 曲线 $(\mathrm{c})$ 为外加磁场垂直 $\left(H_{\perp}\right)$ 于孔轴线方向 的镍微米管的磁滞回线, 其矫顽力为 $H_{\mathrm{c} \perp}=9071 \mathrm{~A} / \mathrm{m}$, $\left(M_{\mathrm{r}} / M_{\mathrm{s}}\right)_{\perp}=0.12$ 。另外，图 4 曲线 $(\mathrm{b})$ 的 $\left(M_{\mathrm{r}} / M_{\mathrm{s}}\right)_{/ /}=0.19$ 。 对比图 4 曲线(b)和(c)结果表明, 外磁场平行和垂直 镍微米管时磁化难易程度相当。但是，垂直于微米 管方向的磁饱和场远大于平行于微米管方向的磁饱 和场，这表明镍微米管有强的各向异性。一般地，铁 磁体的磁矩分布主要取决于磁晶各向异性能、磁弹 性能和退磁能。当磁场平行和垂直于微米管方向时, 磁弹性能应该相同，即各向异性来源于磁晶各向异 性和形状各向异性。当磁场平行和垂直微米管时, 退磁因子相差很大, 分别约为 0.06 和 0.94 。那么, 最 大的退磁能可以表示为:

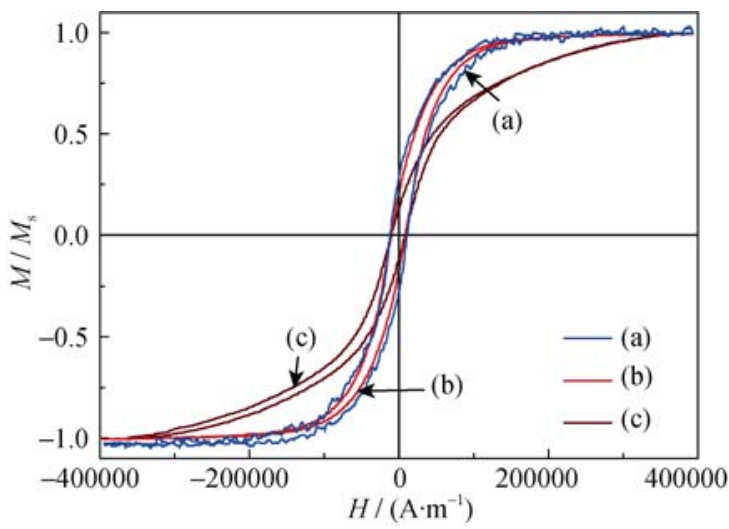

图 4 外加磁场平行和垂直于孔轴线方向的镍颗粒与镍微米 管的磁滞回线

Fig. 4 Magnetic hysteresis loops of Ni particles and microtubes when the external field is parallel or perpendicular to the pore axis

(a)Magnetic hysteresis loops of Ni particles (parallel); (b) Magnetic hysteresis loops of Ni microtube (parallel); (c) Magnetic hysteresis loops of Ni microtube (perpendicular)

$$
E_{\mathrm{d}}(\max )=\frac{1}{2} \times(4 \pi \times 0.94) \times M_{\mathrm{s}}^{2}
$$

其中 $M_{\mathrm{s}}$ 取块材饱和磁化强度 ${ }^{[35]}$ 。由此法估计的 $\mathrm{Ni}$ 微米管退磁能为 $8.55 \times 10^{4} \mathrm{~J} / \mathrm{m}^{3}$, 远大于块体且结晶 完好的镍的磁晶各向异性能 $\left(5.48 \times 10^{3} \mathrm{~J} / \mathrm{m}^{3[35]}\right)$ 。因 此，退磁能是影响镍微米管磁矩分布的主要因素, 造成了镍微米管的磁各向异性。

\section{3 结论}

通过电沉积法在 $\mathrm{N}$ 型多孔硅模板中沉积制备出 了磁性镍微米管。首先利用背光照辅助电化学腐蚀 的方法，在 $\mathrm{N}$ 型轻掺杂的(100)单晶硅上制备了多孔 硅模板。模板孔端口呈近似正方形结构，孔边长约 为 $2 \mu \mathrm{m}$, 孔深约 $50 \mu \mathrm{m}$ 。其中, 模板的生长有成核、 自组装和稳定生长三个阶段。然后利用该模板在不 加背光照的情况下电沉积制备出了镍微米管。随着 沉积时间的延长，镍沿着孔壁均匀生长，由颗粒状 逐渐生长为微米管，且晶型没有变化。磁性能的研 究结果表明：由于镍微米管的缺陷和晶界比镍颗粒 更少，畴壁位移更容易，所以具有更大的矫顽力; 同时，受强烈的退磁能影响，镍微米管表现出明显 的磁各向异性。

\section{参考文献:}

[1] ZHANG HUA, HU YAO-JUAN, WU PING, et al. Preparation of an ultrahigh aspect ratio anodic aluminum oxide template for the fabrication of Ni nanowire arrays. Acta Phys. -Chim. Sin., 2012, 28(6): $1545-1550$

[2] ZHANG DAI-HUA, LIU ZU-QIN, HAN SONG, et al. Magnetite $\left(\mathrm{Fe}_{3} \mathrm{O}_{4}\right)$ core-shell nanowires: synthesis and magnetoresistance. Nano Lett., 2004, 4(11): 2151-2155.

[3] LIAO ZHI-MIN, LI YA-DONG, XU JUN, et al. Spin-filter effect in magnetite nanowire. Nano Lett., 2006, 6(6): 1087-1091.

[4] LI YANG, XIE HUA-QING, LI JING. Hydrothermal synthesis of Al-doped $\alpha-\mathrm{MnO}_{2}$ nanotubes and their electrochemical performance for supercapacitors. Acta Phys. -Chim. Sin., 2015, 31(4): 693-699.

[5] NARAYANAN T N, SANDEEP C S, SHAIJUMON M M, et al. The synthesis of high coercivity cobalt-in-carbon nanotube hybrid structures and their optical limiting properties. Nanotechnology, 2009, 20(28): 095702.

[6] HAN X F, SHAMAILA S, SHARIF R, et al. Structural and magnetic properties of various ferromagnetic nanotubes. Adv. Mater., 2009, 21(45): 1-6.

[7] KARTOPU G, YALCIN O, ES-SOUNI M, et al. Magnetization behavior of ordered and high density Co nanowire arrays with varying aspect ratio. J. Appl. Phys., 2008, 103(9): 093915.

[8] SHIN Y S, HONG J Y, RYU D H, et al. The role of $\mathrm{H}_{2}$ in the growth of carbon nanotubes on an AAO template. J. Korean Phys. Soc., 2007, 50(4): 1068-1072.

[9] LEHMANN V, FÖLL H. Formation mechanism and properties of electrochemically etched trenches in n-type silicon. J. Electrochem. Soc., 1990, 137(2): 653-659. 
[10] VAN DEN MEERAKKER J E A M, ELFRINK R J G, ROOZEBOOM F, et al. Etching of deep macropores in 6 in. $\mathrm{Si}$ wafers. J. Electrochem. Soc., 2000, 147(7): 2757-2761.

[11] BADEL X, RAJENDRA KUMAR R T, KLEIMANN P, et al. Formation of ordered pore arrays at the nanoscale by electrochemical etching of n-type silicon. Superlattices Microstruct, 2004, 36(1/2/3): 245-253.

[12] KLEIMANN P, LINNROS J, PETERSSON S. Formation of wide and deep pores in silicon by electrochemical etching. Mater. Sci. Eng. B, 2000, 69-70: 29-33.

[13] LINNROS J, BADEL X, KLEIMANN P. Macropore and pillar array formationin silicon by electrochemical etching. Phys. Scr., 2006, T126: 72-76.

[14] OTTOW S, LEHMANN V, FÖLL H. Processing of three-dimensional microstructures using macroporous n-type silicon. J. Electrochem. Soc., 1996, 143(1): 385-390.

[15] LEHMANN V. The physics of macropore formation in low doped n-type silicon. J. Electrochem. Soc., 1993, 140(10): 2836-2843.

[16] FÖLL H, CHRISTOPHERSEN M, CARSTENSEN J, et al. Formation and application of porous silicon. Mater. Sci. Eng. R, 2002, 39(4): 93-141.

[17] KOROTCENKOV G, CHO B K. Silicon porosification: state of the art. Crit. Rev. Solid State Mater. Sci., 2010, 35(3): 153-260.

[18] OUIR S, SAM S, FORTAS G, et al. FeNi alloys electroplated into porous(n-type)silicon. Phys. Status Solidi C, 2008, 5(12): 3694-3697.

[19] RONKEL F, SCHULTZE J W, ARENS-FISHER R. Electrical contact to porous silicon by electrodeposition of iron. Thin Solid Film, 1996, 276: 40-43.

[20] RENAUX C, SCHEUREN V, FLANDRE D. New experiments on the electrodeposition of iron in porous silicon. Microelectron. Reliab, 2000, 40(4/5): 877-879.

[21] ARAVAmudhan S, LUONGO K, PODDAR P, et al. Porous silicon templates for electrodeposition of nanostructures. Appl. Phys. A, 2007, 87(4): 773-780.

[22] RUMPF K, GRANITZER P, ALBU M, et al. Electrochemically fabricated silicon/metal hybrid nanosystem with tailored magnetic properties. Electrochem. Solid-State Lett., 2010, 13(2): K15-K18.

[23] HARRAZ F A, SALEM A M, MOHAMED B A, et al. Electrochemically deposited cobalt/platinum(Co/Pt)film into porous sili- con: structural investigation and magnetic properties. Appl. Surf. Sci., 2013, 264: 391-398.

[24] BANDARENKA H, BALUCANI M, CRESCENZI R, et al. Formation of composite nanostructures by corrosive deposition of copper into porous silicon. Superlattices Microstruct., 2008, 44(4/5): 583-587.

[25] FUKAMI K, TANAKA Y, CHOUROU M L, et al. Filling of mesoporous silicon with copper by electrodeposition from an aqueous solution. Electrochim. Acta, 2009, 54(8): 2197-2202.

[26] GRANITZER P, RUMPF K, OHTA T, et al. Porous silicon/Ni composites of high coercivity due to magnetic field-assisted etching. Nanoscale Res. Lett., 2012, 7(1): 384.

[27] GRANITZER P, RUMPF K, POLT P, et al. Self-assembled mesoporous silicon in the crossover between irregular and regular arrangement applicable for Ni filling. Physica E, 2007, 38(1/2): 205-210.

[28] FANG C, FOCA E, XU S, et al. Deep silicon macropores filled with copper by electrodeposition. J. Electrochem. Soc., 2007, 154(1): D45-D49.

[29] FUKAMI K, KOBAYASHI K, MATSUMOTO T, et al. Electrodeposition of noble metals into ordered macropores in p-type silicon. J. Electrochem. Soc., 2008, 155(1): D443-D448.

[30] KOBAYASHI K, HARRAZ F A, IZUO S, et al. Microrod and microtube formation by electrodeposition of metal into ordered macropores prepared in p-type silicon. J. Electrochem. Soc., 2006, 153(4): C218-C222.

[31] SATO H, HOMMA T. Fabrication of high-aspect-ratio arrayed structures using Si electrochemical etching. Sci. Technol. Adv. Mater., 2006, 7(5): 468-474.

[32] HEJJO AL RIFAI M, CHRISTOPHERSEN M, OTTOW S, et al. Dependence of macropore formation in $\mathrm{n}-\mathrm{Si}$ on potential, temperature, and doping. J. Electrochem. Soc., 2000, 147(2): 627-635.

[33] WANG XUE-WEI, YUAN ZHI-HAO, FANG BING-CHENG. Template-based synthesis and magnetic properties of Ni nanotube arrays with different diameters. Mater. Chem. Phys., 2011, 125(1/2): 1-4.

[34] GONG WEI, LI HUA, ZHAO ZHONG-REN, et al. Ultrafine particles of Fe, Co, and Ni ferromagnetic metals. J. Appl. Phys., 1991, 69(8): 5119-5121.

[35] 钟文定. 铁磁学. 北京: 科学出版社, 1998: 7-15. 